\title{
Use of Risk Models to Predict Death in the Next Year Among Individual Ambulatory Patients With Heart Failure
}

\author{
Larry A. Allen, MD, MHS; Daniel D. Matlock, MD, MPH; Susan M. Shetterly, MS; Stanley Xu, PhD; \\ Wayne C. Levy, MD; Laura B. Portalupi, MSW; Colleen K. Mcllvennan, DNP, ANP; Jerry H. Gurwitz, MD; \\ Eric S. Johnson, PhD, MPH; David H. Smith, RPh, MHA, PhD; David J. Magid, MD, MPH
}

IMPORTANCE The clinical practice guidelines for heart failure recommend the use of validated risk models to estimate prognosis. Understanding how well models identify individuals who will die in the next year informs decision making for advanced treatments and hospice.

OBJECTIVE To quantify how risk models calculated in routine practice estimate more than $50 \%$ 1-year mortality among ambulatory patients with heart failure who die in the subsequent year.

DESIGN, SETTING, AND PARTICIPANTS Ambulatory adults with heart failure from 3 integrated health systems were enrolled between 2005 and 2008. The probability of death was estimated using the Seattle Heart Failure Model (SHFM) and the Meta-Analysis Global Group in Chronic Heart Failure (MAGGIC) risk calculator. Baseline covariates were collected from electronic health records. Missing covariates were imputed. Estimated mortality was compared with actual mortality at both population and individual levels.

MAIN OUTCOMES AND MEASURES One-year mortality.

RESULTS Among 10930 patients with heart failure, the median age was 77 years, and $48.0 \%$ of these patients were female. In the year after study enrollment, 1661 patients died (15.9\% by life-table analysis). At the population level, 1-year predicted mortality among the cohort was $9.7 \%$ for the SHFM (C statistic of 0.66 ) and $17.5 \%$ for the MAGGIC risk calculator (C statistic of 0.69). At the individual level, the SHFM predicted a more than $50 \%$ probability of dying in the next year for 8 of the 1661 patients who died (sensitivity for 1-year death was $0.5 \%$ ) and for 5 patients who lived at least a year (positive predictive value, $61.5 \%$ ). The MAGGIC risk calculator predicted a more than $50 \%$ probability of dying in the next year for 52 of the 1661 patients who died (sensitivity, 3.1\%) and for 63 patients who lived at least a year (positive predictive value, $45.2 \%$ ). Conversely, the SHFM estimated that 8496 patients (77.8\%) had a less than $15 \%$ probability of dying at 1 year, yet this lower-risk end of the score range captured nearly two-thirds of deaths $(n=997)$; similarly, the MAGGIC risk calculator estimated a probability of dying of less than $25 \%$ for the majority of patients who died at 1 year $(n=914)$.

CONCLUSIONS AND RELEVANCE Although heart failure risk models perform reasonably well at the population level, they do not reliably predict which individual patients will die in the next year.

JAMA Cardiol. 2017;2(4):435-441. doi:10.1001/jamacardio.2016.5036

Published online December 21, 2016.
Author Affiliations: Author affiliations are listed at the end of this article.

Corresponding Author: Larry A Allen, MD, MHS, Division of Cardiology, University of Colorado School of Medicine, Academic Office 1, Room 7019, 12631E 17th Ave, Mail Stop B130, Aurora, CO 80045 (larry.allen@ucdenver.edu). 
A $\mathrm{n}$ accurate assessment of short-term mortality is central to the care of patients with heart failure. Implantable cardioverter-defibrillators are reserved for patients "who have a reasonable expectation of meaningful survival for more than 1 year.”1(pe183) Left ventricular assist devices and transplantation are restricted to patients whose 1-year mortality without surgery exceeds the expected mortality with surgery (approximately $20 \%$ among patients with a durable left ventricular assist device). ${ }^{2}$ Hospice eligibility relies on a physician's certification "that the patient's life expectancy is 6 months or less."2(p32 176)

Guidelines recommend using validated risk scores to estimate the risk of mortality for patients with heart failure, ${ }^{1}$ and those "who are at high-risk for 1-year mortality using prognostic models should be referred for advanced therapy." ${ }^{\text {(p159) }}$ The Seattle Heart Failure Model (SHFM) is well-validated, ${ }^{4-6} \mathrm{com}$ monly used, and available online (https://depts.washington .edu/shfm/). ${ }^{7}$ The Meta-Analysis Global Group in Chronic Heart Failure (MAGGIC) risk calculator is validated, increasingly used, and available online (http://www.heartfailurerisk.org). ${ }^{8}$ Yet, the application of population-risk data to individual treatment decisions has rarely been characterized. ${ }^{9,10}$ We compared the SHFM and MAGGIC risk calculator estimates with actual deaths at 1 year among a cohort of ambulatory patients with heart failure, for the overall population and for individual patients.

\section{Methods}

All ambulatory patients 21 years of age or older with a diagnosis of heart failure ${ }^{11,12}$ during the period from 2005 to 2008 were identified from Kaiser Permanente Colorado, Kaiser Permanente Northwest, and Fallon Health. Baseline covariates for the risk calculators (Table 1) were extracted from electronic health records. Deaths were identified from health plan databases, state death certificates, and Social Security Administration files. ${ }^{13}$

The SHFM and MAGGIC risk calculator scores were calculated using the online algorithms. ${ }^{7,8}$ Mimicking the calculators, we imputed the mean values for missing data. New York Heart Association functional class, available in routine care but unavailable in electronic records, was set to functional class III in primary analysis and class IV in secondary analysis. To address concerns about model transportability, we updated the intercept and parameter estimates. The SHFM scores were converted to estimated survival at specific times using the following function: survival $(t)=\exp (-0.0405 t)^{e(\text { SHFM score })}$, where $t$ is time. The MAGGIC risk calculator estimates for mortality were mapped based on probabilities for the integer scores 0 to 50 , as described in the original derivation. ${ }^{8}$ Following the published method, ${ }^{8}$ we used multiple imputation for the left ventricular ejection fraction, body mass index, systolic blood pressure, serum creatinine level, and smoking status. The MAGGIC risk calculator uses the left ventricular ejection fraction in interactions with age and systolic blood pressure, so we performed a secondary analysis restricted to persons with left ventricular ejection fraction data. Actual survival was described using life-table analyses to handle truncated

\section{Key Points}

Question How well do heart failure risk models perform in identifying which patients will die in the next year?

Findings In this cohort study of 10930 adults with heart failure, 1661 patients died in the next year. One risk model predicted a more than $50 \%$ probability of dying for only 8 of those patients, and another risk model predicted death for only 52 of those patients.

Meaning Heart failure risk models perform reasonably well at the population level, but they do not reliably predict which individual patients will die in the next year.

follow-up. ${ }^{14}$ The institutional review boards at the 3 participating sites approved the study, and waiver of consent was obtained because of the nature of the study.

\section{Results}

Among 10930 patients with heart failure, the median age was 77 years, and $48.0 \%$ of these patients were female (Table 1 ). In the year following registry inclusion, 1661 patients died (15.9\% by life-table analysis).

Reflecting naturalistic data capture, $75 \%$ of the patients had at least 15 of the 20 SHFM covariates (range, 11-19 covariates). At the population level, the SHFM estimated that the 1-year mortality rate was $9.7 \%$, with a median life expectancy of 7.0 years (interquartile range, 5.3-9.0 years; range, 3 months [shortest predicted remaining survival of anyone in the cohort] to 22 years [longest]). Discrimination was similar to other cohorts: the $C$ statistic was 0.69 at 6 months, 0.66 at 1 year, and 0.65 at 3 years. Model calibration showed underestimations: a 6-month predicted mortality rate of 5.0\% vs an observed mortality rate of $10.5 \%$; a 1-year predicted mortality rate of $9.7 \%$ vs an observed mortality rate of $15.9 \%$; and a 3-year predicted mortality rate of $24.6 \%$ vs an observed mortality rate of $33.6 \%$. The Hosmer-Lemeshow test suggested an adequate model fit $\left(\mathrm{X}^{2}=8.7, P=.36\right)$.

At the individual level, the SHFM performed poorly (Figure). The SHFM predicted a more than $50 \%$ mortality rate in the next year for 8 of the 1661 patients who actually died (sensitivity for 1-year death was $0.5 \%$ ) and for 5 patients who lived at least a year (positive predictive value, 61.5\%) (Table 2). In secondary analysis forcing the New York Heart Association to the worst functional class, the SHFM predicted a more than 50\% 1-year mortality rate among 49 patients who died (sensitivity, 3.0\%) and among 47 patients who lived at least a year (positive predictive value, 51.0\%). Updating the SHFM intercept and parameter estimates to recalibrate and refit the model (which could not be done in clinical practice), we found that the $C$ statistic improved (0.75) but still captured less than $8 \%$ of patients who died in the next year (of 191 patients predicted to die, 123 actually died). Conversely, the SHFM estimated that 8496 patients $(77.7 \%$ ) had a more than $85 \%$ probability of surviving 1 year, yet this "lower-risk" end of the score range captured nearly two-thirds of deaths $(n=997)$. 


\begin{tabular}{|c|c|c|c|}
\hline \multirow[b]{2}{*}{ Variable } & \multirow{2}{*}{$\begin{array}{l}\text { Patients, No. (\%) } \\
(\mathrm{N}=10930)\end{array}$} & \multicolumn{2}{|c|}{ Covariate } \\
\hline & & SHFM & MAGGIC \\
\hline Age, mean [SD], y & $75.1[11.8]$ & $x$ & $x$ \\
\hline Male sex & $5681(52.0)$ & $x$ & $x$ \\
\hline NYHA class ${ }^{\mathrm{a}}$ & & $x$ & $x$ \\
\hline Weight, ${ }^{\mathrm{b}}$ mean [SD], kg & $84.8[24.2]$ & $x$ & \\
\hline BMI & & & $x$ \\
\hline$<15$ & $10(0.1)$ & & \\
\hline $15-19$ & $336(3.1)$ & & \\
\hline $20-24$ & $1776(16.2)$ & & \\
\hline $25-29$ & $2629(24.1)$ & & \\
\hline$\geq 30$ & $3480(31.8)$ & & \\
\hline Missing & $2699(24.7)$ & & \\
\hline LVEF category & & $x$ & $x$ \\
\hline Preserved ( $\geq 50 \%$ ) & $4155(38.0)$ & & \\
\hline Borderline (41\%-49\%) & $1330(12.2)$ & & \\
\hline Reduced $(\leq 40 \%)$ & $3019(27.6)$ & & \\
\hline Missing & $2426(22.2)$ & & \\
\hline $\mathrm{SBP}$, mean [SD], mm Hg & $129.2[22.9]$ & $x$ & $x$ \\
\hline$<110$ & $1524(13.9)$ & & \\
\hline $110-119$ & $1389(12.7)$ & & \\
\hline $120-129$ & $1739(15.9)$ & & \\
\hline $130-139$ & $1511(13.8)$ & & \\
\hline $140-149$ & $1203(11.0)$ & & \\
\hline$\geq 150$ & $1503(13.8)$ & & \\
\hline Missing & 2061 (18.9) & & \\
\hline Ischemic etiology & $2239(20.5)$ & $x$ & \\
\hline \multicolumn{4}{|l|}{ Prescription drug use } \\
\hline ACE inhibitor & $4499(41.2)$ & $\mathrm{x}$ & \\
\hline ARB & $1303(11.9)$ & $x$ & \\
\hline ACE inhibitor/ARB & $5705(52.2)$ & & $x$ \\
\hline$\beta$-Blocker & $6374(58.3)$ & $x$ & $x$ \\
\hline Statin & $4734(43.3)$ & $x$ & \\
\hline Allopurinol & $637(5.8)$ & $x$ & \\
\hline Aldosterone blocker & $1106(10.1)$ & $x$ & \\
\hline $\begin{array}{l}\text { Furosemide equivalents, }{ }^{\mathrm{C}} \mathrm{mg} \\
\text { (24-h dose) }\end{array}$ & & $x$ & \\
\hline No loop diuretic $R x$ & $5471(50.1)$ & & \\
\hline$<20$ & $16(0.1)$ & & \\
\hline 20 & 2287 (20.9) & & \\
\hline $27^{d}$ & $127(1.2)$ & & \\
\hline 40 & $2474(22.6)$ & & \\
\hline $53^{d}$ & $156(1.4)$ & & \\
\hline 80 & $286(2.6)$ & & \\
\hline 100 & $84(0.8)$ & & \\
\hline 200 & $29(0.3)$ & & \\
\hline
\end{tabular}




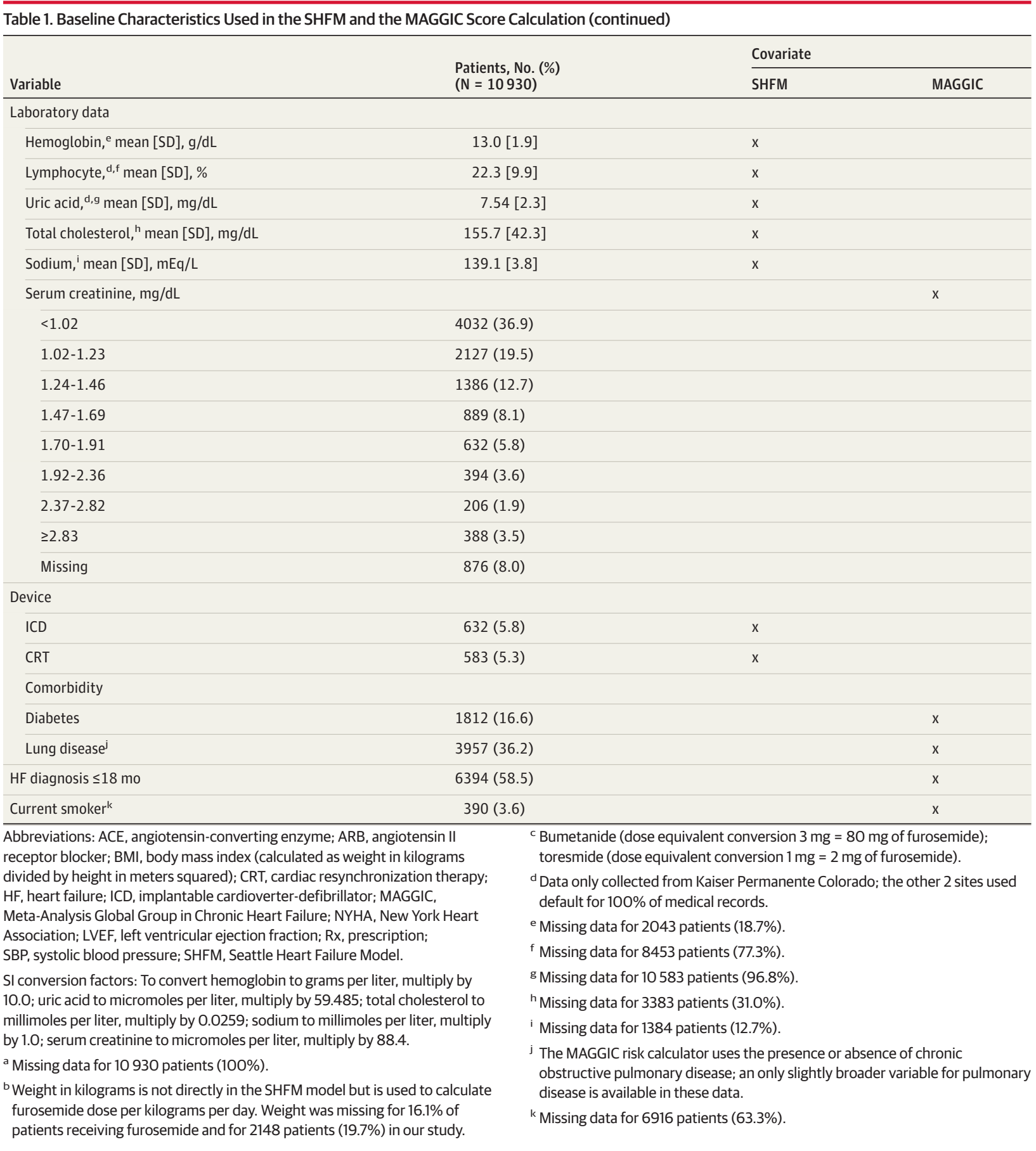

For the more stringent hospice referral threshold, the SHFM identified only 2 patients with a more than $50 \%$ probability of dying within 6 months, neither of whom died (5 patients had a New York Heart Association functional class set to IV). For a lower threshold of less than 20\% 1-year mortality (which can guide a durable left ventricular assist device), the SHFM showed a sensitivity of $20.7 \%$, a specificity of $93.1 \%$, a positive predictive value of $39.6 \%$, and a negative predictive value of $84.4 \%$.

Analysis was repeated using the MAGGIC risk calculator. At the population level, the median score was 26 with a pre- dicted 1-year mortality rate of $17.5 \%$. The $\mathrm{C}$ statistic at 1 year was 0.69. The Hosmer-Lemeshow statistic had a $x^{2}$ of 38.6 $(P<.001)$, indicating some lack of fit in this population. In secondary analyses among the 8504 patients with available left ventricular ejection fraction data, overall risk of death was lower (1150 died [13.5\%]), the $C$ statistic was 0.70, and the Hosmer-Lemeshow $\chi^{2}$ was $16.9(P=.02)$.

At the individual level, the MAGGIC risk calculator also performed poorly (Figure). It predicted a more than $50 \%$ mortality rate in the next year for 52 of the 1661 patients who actually died (sensitivity, 3.1\%) and for 63 patients who lived 
B MAGGIC-predicted 1-y estimated risk of death
Accurate predication for individuals

Inaccurate predication for individuals
SHFM-Predicted Mortality at 1 y

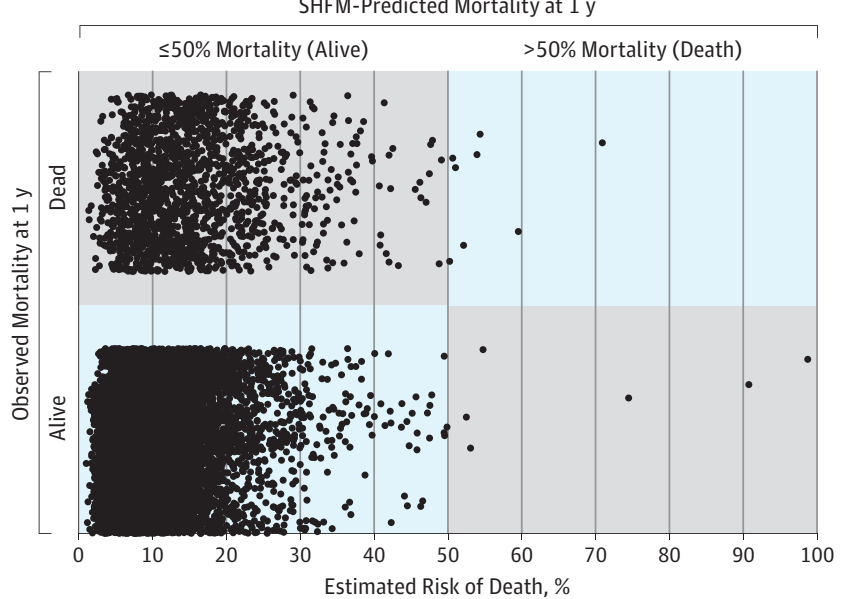

MAGGIC-Predicted Mortality at $1 \mathrm{y}$

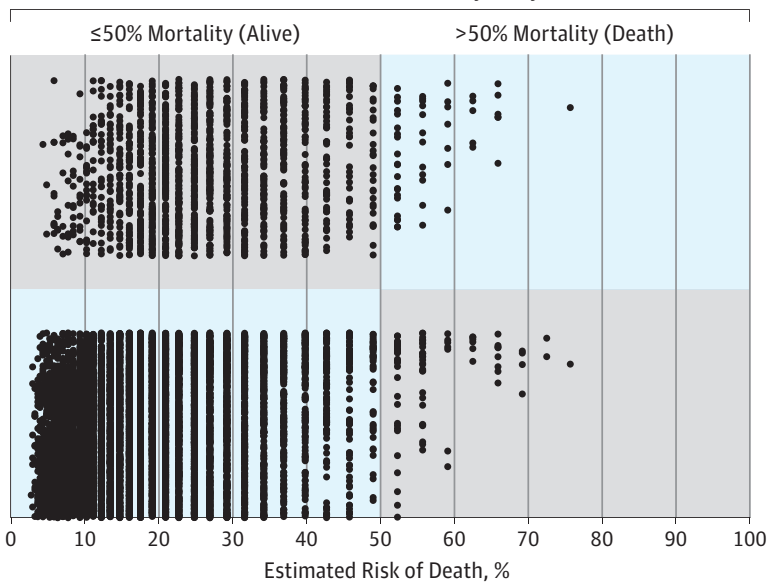

Shown are the 1-year predictions of death for individuals and associated actual survival using the Seattle Heart Failure Model (SHFM) (A) and the Meta-Analysis Global Group in Chronic Heart Failure (MAGGIC) risk calculator (B). Each dot represents an individual.

\begin{tabular}{|c|c|c|c|}
\hline \multirow[b]{2}{*}{ Model } & \multicolumn{2}{|l|}{ 1-y Vital Status } & \multirow[b]{2}{*}{ No./Total No. (\%) } \\
\hline & Died & Lived & \\
\hline \multicolumn{4}{|l|}{ SHFM } \\
\hline Predicted survival $\leq 50 \%$ (anticipated dead), No. & 8 & 5 & $8 / 13(61.5)^{a}$ \\
\hline Predicted survival >50\% (anticipated alive), No. & 1653 & 7616 & $7616 / 9269(82.2)^{b}$ \\
\hline No./total No. (\%) & $\begin{array}{l}8 / 1661 \\
(0.5)^{c}\end{array}$ & $\begin{array}{c}7616 / 7621 \\
(99.9)^{\mathrm{d}}\end{array}$ & $9282^{\mathrm{e}}$ \\
\hline \multicolumn{4}{|l|}{ MAGGIC risk calculator } \\
\hline Predicted survival $\leq 50 \%$ (anticipated dead), No. & 52 & 63 & $52 / 115(45.2)^{\mathrm{a}}$ \\
\hline Predicted survival >50\% (anticipated alive), No. & 1609 & 7558 & $7558 / 9167(82.4)^{b}$ \\
\hline No./total No. (\%) & $52 / 1661(3.1)^{c}$ & $7558 / 7621(99.2)^{d}$ & $9282^{\mathrm{e}}$ \\
\hline
\end{tabular}

Abbreviations: MAGGIC, Meta-Analysis Global Group in Chronic Heart Failure; SHFM, Seattle Heart Failure Model.

a Positive predictive value.

${ }^{\mathrm{b}}$ Negative predictive value. c Sensitivity.

d Specificity.

e A total of 1648 patients were censored owing to a lack of 1-year follow-up ( 25 patients with a MAGGIC-predicted survival of $\leq 50 \%$ ). at least a year (positive predictive value, 45.2\%) (Table 2). Conversely, the MAGGIC risk calculator estimated that 8161 patients $(74.7 \%)$ had a more than $75 \%$ probability of surviving 1 year, yet this "lower-risk" end of the score range captured the majority of deaths $(n=914)$. For the lower threshold of less than 20\% 1-year mortality, the MAGGIC risk calculator showed a sensitivity of $69.7 \%$, a specificity of $61.2 \%$, a positive predictive value of $28.1 \%$, and a negative predictive value of $90.3 \%$.

\section{Discussion}

In this cohort of more than 10000 ambulatory patients with heart failure, well-accepted risk models assigned a more than $50 \%$ chance of death in the next year to a very small fraction of those who actually died. Conversely, the majority of patients who died had a more than $75 \%$ model-estimated probability of surviving beyond 1 year. This highlights the limita- tions of applying population-based prognostic model results to short-term medical decisions about individual patients ${ }^{15,16}$ and may partially justify the limited uptake of risk models in routine clinical practice. ${ }^{17}$

Most research has gone into refining risk models despite diminishing improvements in performance. Meanwhile, there is a dearth of information to help patients and health care professionals manage the uncertainty inherent in survival estimates. ${ }^{18}$ Objective risk information should be integrated with recognition of uncertainty. ${ }^{15,19,20}$ For example, prediction intervals may be more informative than median values. ${ }^{21}$ Rather than say that "your expected survival is about 1 year," a clinician could say "if I had 100 people like you, 25 would die within 6 months and 25 would be alive after 2 years."

\section{Limitations}

Limitations should be considered. First, missing data requiring imputation may significantly relate to the poor risk pre- 
diction seen in these models; however, imputation replicates most real-world risk assessments. Second, the model calculation was performed some years ago, but the rates of therapy and mortality for heart failure remained relatively unchanged from 2005 to $2014 .^{22,23}$ Finally, the risk models were developed in cohorts that either reflected secular differences in time (the SHFM) or geographical differences in care (the MAGGIC risk calculator). Moreover, the cohort used herelike any insured population in an integrated health planmay fail to track well with the general population. Variability in the performance of the SFHM and the MAGGIC risk calculator is to be expected because the derivation cohorts came from highly orchestrated clinical trial populations instead of unselected general care patients.

\section{Conclusions}

Risk models cannot prospectively identify the vast majority of individual ambulatory patients who will die in the near future. For undulating diseases such as heart failure, improvements in risk modeling are only a partial solution. Meanwhile, we must embrace the certainty of uncertainty and better help patients plan for the worst while hoping for the best.

\section{ARTICLE INFORMATION}

Accepted for Publication: October 28, 2016.

Published Online: December 21, 2016. doi:10.1001/jamacardio.2016.5036

Author Affiliations: Division of Cardiology, University of Colorado School of Medicine, Aurora (Allen, Mcllvennan); Institute for Health Research, Kaiser Permanente Colorado, Denver (Allen, Matlock, Shetterly, Xu); Adult and Child Consortium for Outcomes Research and Delivery Science University of Colorado School of Medicine, Aurora (Allen, Matlock, Portalupi, Mcllvennan); Division of Geriatrics, University of Colorado School of Medicine, Aurora (Matlock); University of Washington School of Medicine, Seattle (Levy); Division of Geriatric Medicine, University of Massachusetts Medical School, Worcester (Gurwitz); Meyers Primary Care Institute, a joint endeavor of the University of Massachusetts Medical School, Fallon Health, and the Reliant Medical Group, Worcester (Gurwitz); Kaiser Permanente Center for Health Research, Portland, Oregon (Johnson, Smith); Colorado Permanente Medical Group, Denver (Magid)

Author Contributions: Dr Allen had full access to all of the data in the study and takes responsibility for the integrity of the data and the accuracy of the data analysis.

Concept and design: Allen, Matlock, Xu, Levy, Mcllvennan, Johnson.

Acquisition, analysis, or interpretation of data: Allen Shetterly, Xu, Portalupi, Gurwitz, Johnson, Smith Magid.

Drafting of the manuscript: Allen. Critical revision of the manuscript for important intellectual content: Matlock, Shetterly, Xu, Levy, Portalupi, Mcllvennan, Gurwitz, Johnson, Smith, Magid.

Statistical analysis: Shetterly, Xu, Johnson. Obtained funding: Allen, Gurwitz.

Administrative, technical, or material support: Allen, Levy, Portalupi, Mcllvennan, Gurwitz, Smith. Study supervision: Shetterly, Gurwitz.

Conflict of Interest Disclosures: All authors have completed and submitted the ICMJE Form for Disclosure of Potential Conflicts of Interest. Dr Allen receives grant funding from the National Institutes of Health and the Patient-Centered Outcomes Research Institute and consultancy fees from Novartis, Janssen, St Jude, and ZS Pharma. Dr Levy developed the SHFM. No other disclosures are reported.

Funding/Support: Dr Allen's time and the analytics for this study were supported by grant
K23HL105896 from the National Heart, Lung, and Blood Institute of the National Institutes of Health. Additional support for cohort creation was provided by the American Recovery and Reinvestment Act grant 1RC1HL099395 (the PRESERVE [Predicting the Safety and Effectiveness of Inferior Vena Cava Filters] study) administered through the National Heart, Lung, and Blood Institute. Dr Matlock was supported by grant K23AG040696 from the National Institute on Aging

Role of the Funder/Sponsor: The funders/ sponsors had no role in the design and conduct of the study; collection, management, analysis, and interpretation of the data; preparation, review, or approval of the manuscript; and decision to submit the manuscript for publication.

Additional Contributions: We wish to thank all of the project managers, data programmers, and analysts for their critical technical contributions and support that made this study possible.

\section{REFERENCES}

1. Yancy CW, Jessup $M$, Bozkurt B, et al; American College of Cardiology Foundation; American Heart Association Task Force on Practice Guidelines. 2013 ACCF/AHA guideline for the management of heart failure: a report of the American College of Cardiology Foundation/American Heart Association Task Force on Practice Guidelines. J Am Coll Cardiol. 2013;62(16):e147-e239.

2. Centers for Medicare \& Medicaid Services (CMS) HHS. Medicare and Medicaid programs: hospice conditions of participation: final rule. Fed Regist. 2008;73(109):32087-32220.

3. Feldman D, Pamboukian SV, Teuteberg JJ, et al International Society for Heart and Lung Transplantation. The 2013 International Society for Heart and Lung Transplantation Guidelines for mechanical circulatory support: executive summary. J Heart Lung Transplant. 2013;32(2): 157-187.

4. Alba AC, Agoritsas T, Jankowski M, et al. Risk prediction models for mortality in ambulatory patients with heart failure: a systematic review. Circ Heart Fail. 2013;6(5):881-889.

5. May HT, Horne BD, Levy WC, et al. Validation of the Seattle Heart Failure Model in a community-based heart failure population and enhancement by adding B-type natriuretic peptide. Am J Cardiol. 2007;100(4):697-700.

6. Kalogeropoulos AP, Georgiopoulou VV, Giamouzis G, et al. Utility of the Seattle Heart
Failure Model in patients with advanced heart failure. J Am Coll Cardiol. 2009;53(4) 334-342.

7. Levy WC, Mozaffarian D, Linker DT, et al. The Seattle Heart Failure Model: prediction of survival in heart failure. Circulation. 2006;113(11): 1424-1433.

8. Pocock SJ, Ariti CA, McMurray JJ, et al; Meta-Analysis Global Group in Chronic Heart Failure. Predicting survival in heart failure: a risk score based on 39372 patients from 30 studies. Eur Heart J. 2013;34(19):1404-1413.

9. O'Callaghan A, Laking G, Frey R, Robinson J, Gott M. Can we predict which hospitalised patients are in their last year of life? a prospective cross-sectional study of the Gold Standards Framework Prognostic Indicator Guidance as a screening tool in the acute hospital setting. Palliat Med. 2014;28(8): 1046-1052.

10. Haga K, Murray S, Reid J, et al. Identifying community based chronic heart failure patients in the last year of life: a comparison of the Gold Standards Framework Prognostic Indicator Guide and the Seattle Heart Failure Model. Heart. 2012;98 (7):579-583.

11. Go AS, Magid DJ, Wells B, et al. The Cardiovascular Research Network: a new paradigm for cardiovascular quality and outcomes research. Circ Cardiovasc Qual Outcomes. 2008:1(2) 138-147.

12. Go AS, Yang J, Gurwitz JH, Hsu J, Lane K, Platt R. Comparative effectiveness of different beta-adrenergic antagonists on mortality among adults with heart failure in clinical practice. Arch Intern Med. 2008;168(22):2415-2421.

13. Go AS, Yang J, Ackerson LM, et al. Hemoglobin level, chronic kidney disease, and the risks of death and hospitalization in adults with chronic heart failure: the Anemia in Chronic Heart Failure: Outcomes and Resource Utilization (ANCHOR) Study. Circulation. 2006;113(23): 2713-2723.

14. Collett D. Modelling Survival Data in Medical Research. New York, NY: Chapman \& Hall; 1994.

15. Politi MC, Han PK, Col NF. Communicating the uncertainty of harms and benefits of medical interventions. Med Decis Making. 2007;27(5): 681-695.

16. Kent DM, Shah ND. Risk models and patient-centered evidence: should physicians expect one right answer? JAMA. 2012;307(15): 1585-1586. 
17. Steyerberg EW, Moons KGM, van der Windt DA, et al; PROGRESS Group. Prognosis Research Strategy (PROGRESS) 3: prognostic model research. PLoS Med. 2013;10(2):e1001381.

18. Smith AK, White DB, Arnold RM.

Uncertainty-the other side of prognosis. N Engl J Med. 2013;368(26):2448-2450.

19. Henderson R, Jones M, Stare J. Accuracy of point predictions in survival analysis. Stat Med. 2001;20(20):3083-3096.
20. Henderson R, Keiding N. Individual survival time prediction using statistical models. J Med Ethics. 2005;31(12):703-706.

21. Olive DJ. Prediction intervals for regression models. Comput Stat Data Anal. 2007;51(6):31153122. doi:10.1016/j.csda.2006.02.006

22. Sacks CA, Jarcho JA, Curfman GD. Paradigm shifts in heart-failure therapy-a timeline. N Engl J Med. 2014;371(11):989-991.
23. Mozaffarian D, Benjamin EJ, Go AS, et al; American Heart Association Statistics Committee and Stroke Statistics Subcommittee. Heart disease and stroke statistics-2015 update: a report from the American Heart Association. Circulation. 2015; 131(4):e29-e322. 